BULLETIN (New Series) OF THE

Volume 35, Number 2, April 1998, Pages 105-122

S 0273-0979(98)00749-6

\title{
MODULAR REPRESENTATIONS OF SIMPLE LIE ALGEBRAS
}

\author{
J. E. HUMPHREYS
}

To the memory of Boris Weisfeiler

\begin{abstract}
In spite of many efforts over the past 50 years, the irreducible representations of the Lie algebra of a simple algebraic group over a field of prime characteristic are poorly understood. Recent work on quantum groups at a root of unity has provided new impetus for the subject. This article surveys what has been done and what remains to be done.
\end{abstract}

\section{INTRODUCTION}

Finite-dimensional representations of simple Lie algebras over $\mathbb{C}$ have been wellstudied, from a variety of viewpoints: the algebraic "highest weight" theory of E. Cartan, the compact group viewpoint of $\mathrm{H}$. Weyl, the geometric viewpoint of A. Borel, A. Weil, R. Bott, etc. Results for the Lie algebra are essentially interchangeable with results for the corresponding simply connected Lie group or a compact real form.

The groups and Lie algebras over $\mathbb{C}$ have analogues over fields of prime characteristic, such as special linear groups $\mathrm{SL}(n, K)$ and Lie algebras $\mathfrak{s l}(n, K)$. Highest weight representations of the groups also occur naturally in this setting, as shown by C. Chevalley in the late 1950's. Apart from their intrinsic interest, these representations play a key role in the representation theory of finite simple groups of Lie type. In the last decade, new motivation for the "modular" theory has arisen from the study of quantum groups at a root of unity.

Our goal is to give a concise overview of what is (and is not) known about the irreducible representations of the Lie algebra $\mathfrak{g}$ of a simple algebraic group $G$ over an algebraically closed field $K$ of characteristic $p>0$. Besides the "restricted" representations obtained by differentiating representations of $G$, there are many others with no obvious connection to group theory. These have been studied sporadically for over half a century, starting with fundamental work of H. Zassenhaus from 1939 onwards in the general setting of modular Lie algebras: see [81].

Recently a lot of new ideas have been introduced here, which are relevant also to quantum groups at a root of unity. One of the high points has been A. Premet's proof [63] of an old conjecture of V. Kac and B. Weisfeiler giving a lower bound on the $p$-powers dividing dimensions of simple modules. But the main problems remain

Received by the editors June 27, 1996, and in revised form February 24, 1998.

1991 Mathematics Subject Classification. Primary 17B20; Secondary 20 G05.

Key words and phrases. Simple Lie algebra, modular representations.

In preparing this survey I have benefited from extensive correspondence and conversations with Jens Carsten Jantzen, as well as advice from Ivan Mirković and Dmitriy Rumynin.

(C)1998 American Mathematical Society 
almost entirely unsolved: for example, to find the dimensions of all irreducible representations of $\mathfrak{g}$.

The reader is assumed to have some acquaintance with the classical theory of finite-dimensional representations of a semisimple Lie algebra over $\mathbb{C}$. Up to a point, the characteristic $p$ theory has a similar flavor. But it turns out to be far more complicated, involving for example not just the usual Weyl group but also the related affine Weyl group - as realized 25 years ago by Verma [79].

Here we consider only simple Lie algebras of "classical type", leaving aside those of "Cartan type", for which related problems arise (cf. Lin-Nakano [45]).

\section{The Lie Algebra of A Simple Algebraic Group}

As Chevalley showed, the classification of simple algebraic groups over $K$ is essentially the same as the classification of simple Lie groups over $\mathbb{C}$. The groups fall into four infinite families of classical groups (of types $A, B, C, D$ ) along with five exceptional types $\left(E_{6}, E_{7}, E_{8}, F_{4}, G_{2}\right)$. Allowing for finite centers, there may be a number of closely related groups for each type, ranging from simply connected to adjoint. For example, in type $A_{n}$ we have the simply connected group $\mathrm{SL}(n+1, K)$, the adjoint group PGL $(n+1, K)$, and various intermediate quotients corresponding to divisors of $n+1$.

The Lie algebra $\mathfrak{g}$ of such a simple algebraic group $G$ is also a close relative of the simple Lie algebra over $\mathbb{C}$ of corresponding type, being obtained (in the simply connected case) by reduction $\bmod p$ using a Chevalley basis. But $\mathfrak{g}$ is not always simple, and its Killing form is not always nondegenerate: for example, when $\mathfrak{g}=\mathfrak{s l}(n, K)$, the Lie algebra of $n \times n$ matrices of trace 0 , there is a 1-dimensional center consisting of scalar matrices when $p$ divides $n$. Working instead with the full $n \times n$ matrix algebra $\mathfrak{g l}(n, K)$ avoids some technical problems in this case. Other exceptional situations involve $p=2,3,5$ (bad primes for some types), and are worked out thoroughly in Hogeweij [23], Hiss [21].

The reader can safely ignore these complications at first, though of course they eventually have to be addressed. Unless otherwise stated, we take $G$ to be simply connected.

As in characteristic 0, a key structural feature of $\mathfrak{g}$ is its "triangular" decomposition $\mathfrak{g}=\mathfrak{n}^{-} \oplus \mathfrak{h} \oplus \mathfrak{n}^{+}$. Here $\mathfrak{h}$ is a Cartan subalgebra (the Lie algebra of a maximal torus $T$ of $G$ ), while $\mathfrak{n}^{+}$and $\mathfrak{n}^{-}$are the respective sums of positive and negative root spaces. Thus $\operatorname{dim} \mathfrak{g}=r+2 N$, where $r=\operatorname{dim} \mathfrak{h}$ is the rank and $N$ is the number of positive roots. When $\mathfrak{g}=\mathfrak{s l}(n, K)$, the respective subalgebras $\mathfrak{h}, \mathfrak{n}^{+}, \mathfrak{n}^{-}$may be taken to consist of diagonal, upper triangular nilpotent, and lower triangular nilpotent matrices of trace 0 .

In representation theory the Borel subalgebra $\mathfrak{b}=\mathfrak{h} \oplus \mathfrak{n}^{+}$plays an especially important role. Its conjugates under the adjoint action of $G$ are the maximal solvable subalgebras of $\mathfrak{g}$ (except in small cases like $\mathfrak{g}=\mathfrak{s l}(2, K), p=2$ ).

\section{IRREDUCIBLE REPRESENTATIONS}

While the structure of our Lie algebra $\mathfrak{g}$ is at first sight not much different from that of the corresponding Lie algebra over $\mathbb{C}$, there is an added ingredient: a $p$-map $x \mapsto x^{[p]}$, which for a matrix Lie algebra such as $\mathfrak{s l}(n, K)$ is just the associative $p$ th power. The $p$-map arises intrinsically from the description of $\mathfrak{g}$ as an algebra of left invariant derivations of the algebra of regular functions on $G$ : here the $p$ th power 
is again a derivation. We call $\mathfrak{g}$ a restricted Lie algebra or Lie p-algebra. (Some of the results below are in fact valid for restricted Lie algebras in the abstract sense introduced by Jacobson [28], without reference to an algebraic group $G$.)

The $p$-map on $\mathfrak{g}$ has a dramatic effect on the structure of the universal enveloping algebra $U(\mathfrak{g})$. Recall that this infinite-dimensional associative algebra has a Poincaré-Birkhoff-Witt basis made up of monomials in elements of any fixed ordered basis of $\mathfrak{g}$. Representations of $\mathfrak{g}$ over $K$ are essentially the same thing as $U(\mathfrak{g})$-modules.

In characteristic 0 the center $Z(\mathfrak{g})$ of $U(\mathfrak{g})$ is comparatively small: it is isomorphic to the Weyl group invariants in $U(\mathfrak{h})$, a polynomial algebra in $r$ variables. This permits simple $U(\mathfrak{g})$-modules to be infinite-dimensional. But in characteristic $p$ the center is much larger. It is easy to see that for each $x \in \mathfrak{g}$, the element $x^{p}-x^{[p]}$ of $U(\mathfrak{g})$ lies in $Z(\mathfrak{g})$. Methods of Zassenhaus [81] (in the more general context of arbitrary modular Lie algebras) show that these elements generate a copy of the polynomial algebra in $n$ indeterminates, where $n=\operatorname{dim} \mathfrak{g}$ : see $[77, \S 5.2]$. This subalgebra of $Z(\mathfrak{g})$ may be denoted $\mathcal{O}\left(\right.$ or $\left.Z_{0}(\mathfrak{g})\right)$ and called the $p$-center. Moreover, $U(\mathfrak{g})$ is a free $\mathcal{O}$-module of rank $p^{n}$. A more precise description of $Z(\mathfrak{g})$ relative to $\mathcal{O}$ is given by Veldkamp [78]: this involves the Weyl group invariants, but requires some restrictions on $p$.

Because the center of $U(\mathfrak{g})$ is so large, arguments due to Jacobson and Curtis [6] (see also Quillen [67]) show, even for non-restricted $\mathfrak{g}$ :

Theorem. All simple $U(\mathfrak{g})$-modules are finite-dimensional, and their dimensions are bounded by a constant which depends only on $\mathfrak{g}$.

In our situation there is a precise upper bound for this constant: $p^{N}$, where $N$ is the number of positive roots. (See Rudakov [68], where some mild restrictions are made on $p$.)

\section{ReduCED ENVELOPING ALGEBRAS}

Each simple $U(\mathfrak{g})$-module is in fact a module for a finite-dimensional quotient algebra. By Schur's Lemma, elements of $Z(\mathfrak{g})$ act on such a module by scalars. Thus for each $x \in \mathfrak{g}$, the element $x^{p}-x^{[p]}$ acts by a scalar $\xi(x)$. Kac and Weisfeiler observed that $\xi$ is the $p$ th power of a linear functional $\chi \in \mathfrak{g}^{*}$, which may be called the $p$-character of the module.

Fix $\chi \in \mathfrak{g}^{*}$. Let $I_{\chi}$ be the ideal of $U(\mathfrak{g})$ generated by the central elements $x^{p}-x^{[p]}-\chi(x)^{p}$, and set $U_{\chi}(\mathfrak{g}):=U(\mathfrak{g}) / I_{\chi}$. This is the reduced enveloping algebra associated with $\chi$. It may also be described as $U(\mathfrak{g}) \otimes_{\mathcal{O}} K$, with $\mathcal{O}$ acting via $\chi^{p}$ on $K$. Each simple $U(\mathfrak{g})$-module is now a module for a uniquely determined $U_{\chi}(\mathfrak{g})$. These algebras were introduced in Weisfeiler-Kac [80], generalizing the restricted enveloping algebra constructed by Jacobson when $\chi=0$. For the latter we use the notation $U^{[p]}(\mathfrak{g})$ in place of $U_{0}(\mathfrak{g})$. (Other notations found in the literature include $V(\mathfrak{g})$ and $u(\mathfrak{g})$.)

Scrutiny of the construction shows readily:

Theorem. If $\operatorname{dim} \mathfrak{g}=n$, then $\operatorname{dim} U_{\chi}(\mathfrak{g})=p^{n}$. More precisely, if $\left\{x_{1}, \ldots, x_{n}\right\}$ is an ordered basis of $\mathfrak{g}$, then $U_{\chi}(\mathfrak{g})$ has a basis consisting of the cosets of all $x_{1}^{a_{1}} \ldots x_{n}^{a_{n}}$ $\left(0 \leq a_{i}<p\right)$.

The algebras $U_{\chi}(\mathfrak{g})$ are Frobenius algebras, and even "symmetric" in the sense of Nesbitt: for $\chi=0$ this goes back to Berkson [4] and Schue [74]; for arbitrary 
$\chi$ see Strade-Farnsteiner [77, 5.4] and Friedlander-Parshall [17, Prop. 1.2]. As a consequence, the projective cover and injective envelope of a simple $U_{\chi}(\mathfrak{g})$-module are isomorphic.

Restricted enveloping algebras $U^{[p]}(\mathfrak{g})$ inherit the Hopf algebra structure of $U(\mathfrak{g})$ (which is already enough to imply they are Frobenius algebras), while other $U_{\chi}(\mathfrak{g})$ do not. Indeed, the dual of a $U_{\chi}(\mathfrak{g})$-module is well-defined as a $U(\mathfrak{g})$-module, but then becomes a module for $U_{-\chi}(\mathfrak{g})$. Similarly, the tensor product of a $U_{\chi}(\mathfrak{g})$-module with a $U_{\psi}(\mathfrak{g})$-module is a module for $U_{\chi+\psi}(\mathfrak{g})$.

It should be emphasized that a full understanding of the representation theory of $U_{\chi}(\mathfrak{g})$ requires more than the determination of simple modules. Other classes of indecomposable modules - especially projectives - eventually have to be brought into the picture, even if one's main goal is to understand simple modules. See for example Humphreys [24], [25], [27], Lin [43], [44], Nakano-Pollack [54].

\section{Restricted REPRESEntations}

Rational representations of the algebraic group $G$ lead, by differentiation, to restricted representations of $\mathfrak{g}$ : in other words, $U^{[p]}(\mathfrak{g})$-modules. Not every $U^{[p]}(\mathfrak{g})$ module is obtained in this way. But in the reverse direction, early work of Curtis [7] and Steinberg [76] shows how to obtain all irreducible rational representations of $G$ from representations of $\mathfrak{g}$ :

Theorem. Assume $G$ is simply connected.

1. All simple $U^{[p]}(\mathfrak{g})$-modules lift to rational representations of $G$.

2. All irreducible rational representations of $G$ are realized by twisted tensor products of simple $U^{[p]}(\mathfrak{g})$-modules.

Here "twisted" refers to combining a representation of $G$ with powers of the Frobenius endomorphism. In principle these results reduce a significant part of the representation theory of $G$ to that of $\mathfrak{g}$. But in practice, Lie algebra techniques have so far been inadequate to solve the problem.

Jantzen's 1987 book [33] provides a comprehensive foundation for the representation theory of both $G$ and $U^{[p]}(\mathfrak{g})$ (as well as related group schemes). Although we are still far from knowing the simple modules, there are by now a lot of refined techniques for organizing the problem and relating it to work of Kazhdan-Lusztig on affine Hecke algebras: linkage principle, translation functors, etc. More recently, all of this has been further enriched by Lusztig's conjectures relating characteristic $p$ representations to the representation theory of quantum groups at a root of unity: see Andersen [1], Andersen-Jantzen-Soergel [2], and the concluding remarks below.

\section{Highest Weights}

What do simple $U^{[p]}(\mathfrak{g})$-modules actually look like? Even though the precise dimensions of these modules remain unknown in general, their internal structure is not much different from that of their counterparts in characteristic 0 .

We need a little more notation. Since $G$ is assumed to be simply connected, $\mathfrak{g}$ has an easily described basis coming by reduction mod $p$ from a Chevalley basis in characteristic 0 . This includes elements $h_{\alpha}$ spanning $\mathfrak{h}$, where $\alpha$ runs over just the $r=\operatorname{dim} \mathfrak{h}$ simple roots. The subalgebra $\mathfrak{n}^{+}$is a direct sum of 1-dimensional root spaces $K e_{\alpha}$, where $\alpha$ runs over the $N$ positive roots, while $\mathfrak{n}^{-}$is the sum of root 
spaces $K f_{\alpha}$ ( $\alpha$ positive). The triples $\left\{h_{\alpha}, e_{\alpha}, f_{\alpha}\right\}$ span copies of $\mathfrak{s l}(2, K)$, where $h_{\alpha}=\left[e_{\alpha} f_{\alpha}\right]$ for any root $\alpha$.

Now any simple $U^{[p]}(\mathfrak{g})$-module $L$ is the direct sum of weight spaces $L_{\mu}$ for various $\mu \in \mathfrak{h}^{*}$, where

$$
L_{\mu}:=\{v \in L \mid h \cdot v=\mu(h) v \text { for all } h \in \mathfrak{h}\} .
$$

Since $L$ lifts to a representation of $G$, all weights in fact come (by differentiation) from characters of a maximal torus $T$ of $G$ whose Lie algebra is $\mathfrak{h}$. If $X=X(T)$ is the character group of $T$, we write $\lambda$ for both an element of $X$ and its differential in $\mathfrak{h}^{*}$. As in characteristic 0, the Weyl group $W=N_{G}(T) / T$ permutes the weights, with $\operatorname{dim} L_{\mu}=\operatorname{dim} L_{w \mu}$. (But these dimensions are in general smaller than those found in characteristic 0 .)

There is a unique line in $L$ annihilated by all $e_{\alpha}$; it is spanned by a weight vector $v$ of weight $\lambda$. We call $v$ a primitive vector and $\lambda$ the highest weight of $L$, which can now be denoted $L(\lambda)$. One gets other weight vectors spanning $L(\lambda)$ by applying monomials in the $f_{\alpha}$ to $v$. As in the classical theory, the possible highest weights $\lambda$ are "dominant" in $X$ relative to the chosen positive roots when $L(\lambda)$ is viewed as a $G$-module. The coordinates of $\lambda$ relative to fundamental weights are $<p$. But the differential of $\lambda$ in $\mathfrak{h}^{*}$ must be regarded as an element of $X / p X$, so the usual partial ordering of weights is lost. In any case, the $p^{r}$ elements of $X / p X$ parametrize the distinct simple $U^{[p]}(\mathfrak{g})$-modules.

The close analogy here with the classical Cartan-Weyl theory depends on the equations $e_{\alpha}^{p}=0=f_{\alpha}^{p}$ and $h_{\alpha}^{p}=h_{\alpha}$ in $U^{[p]}(\mathfrak{g})$. In other reduced enveloping algebras the situation is subtly different, as we shall see.

\section{NON-RESTRICTED REPRESENTATIONS}

Rational representations of $G$ yield only restricted representations of $\mathfrak{g}$. But there has long been a hope that a full understanding of the non-restricted irreducible representations of $\mathfrak{g}$ might lead (by a process of "deformation") to an understanding of the restricted ones. Unfortunately, it is still difficult to make this idea precise. Meanwhile the study of arbitrary $U(\mathfrak{g})$-modules continues to have intrinsic appealin part because the main problems have resisted attack for so many decades.

Our intention here is to describe a few of the high points, skipping over technical details (especially those involving bad primes). The extensive references below should help to round out the picture. But we especially want to call attention to the work of Zassenhaus [81], Kac-Weisfeiler [80], [39], Friedlander-Parshall [17], [18], [19], Premet [62], [63], [64], [65], and Jantzen [35], [36], [37].

\section{Cohdjoint orbits And Premet's Theorem}

As emphasized by Kac and Weisfeiler [80], [39], the adjoint action of $G$ on $\mathfrak{g}$ (or the coadjoint action on $\mathfrak{g}^{*}$ ) is critical to a deeper understanding of the representations of $U(\mathfrak{g})$. An easy first observation is that $U_{\chi}(\mathfrak{g})$ is isomorphic to $U_{\psi}(\mathfrak{g})$ if $\chi$ and $\psi$ lie in the same $G$-orbit in $\mathfrak{g}^{*}$. So one may hope to simplify matters by working with well-chosen orbit representatives.

But there are deeper connections with the orbit geometry. As in characteristic 0, each coadjoint orbit has even dimension. In their 1971 paper Kac and Weisfeiler[80] conjectured (under very mild assumptions) that, if the orbit of $\chi$ has dimension $2 d$, then $p^{d}$ should divide the dimensions of all $U_{\chi}(\mathfrak{g})$-modules. This was verified for 
$\mathfrak{s l}(n, K)$ when $p>n$ by Friedlander-Parshall $[19,5.2]$. The conjecture has recently been proved in general by Premet [62], [63] with a few restrictions on $p$ and the type of $\mathfrak{g}$. It is enough, for example, to assume that $p$ is good for $G$ and that $\mathfrak{g}$ has a nondegenerate $G$-invariant trace form such as the Killing form.

Theorem. Let $\mathfrak{g}$ satisfy Premet's conditions. If the G-orbit of $\chi$ has dimension $2 d$, then $p^{d}$ divides the dimension of every finite-dimensional $U_{\chi}(\mathfrak{g})$-module.

The crucial case is that of a "nilpotent" $\chi$ ( $(15$ below). Premet's strategy is to locate a $d$-dimensional nilpotent subalgebra $\mathfrak{m}$ of $\mathfrak{g}$ so that each $U_{\chi}(\mathfrak{g})$-module $M$ restricts to a projective $U_{\chi}(\mathfrak{m})$-module. But for well-chosen $\mathfrak{m}$ it can be seen that $U_{\chi}(\mathfrak{m})$ has just one simple module and is the projective cover of this module; so "projective" here means "free". Thus $\operatorname{dim} M$ is a multiple of $\operatorname{dim} U_{\chi}(\mathfrak{m})=p^{d}$. While this strategy is easy to describe, it is quite difficult to implement, in part because there really are exceptions to the theorem in cases such as $\mathfrak{s l}(n, K)$ when $p \mid n$. (For another approach see Premet-Skryabin [66].)

Natural questions are raised by the theorem: Is there always a module of dimension $p^{d}$ ? If so, can it be constructed in some explicit fashion? In all cases investigated so far, including $\mathfrak{g}=\mathfrak{s l}(n, K)$ for $p>n$ (Friedlander-Parshall), the answer to the first question is positive. In his extensive review of [63] in Mathematical Reviews, Kac remarks that he and A. Radul have shown the existence of a module of dimension $p^{d}$ whenever $\chi$ lies in the unique minimal (nonzero) nilpotent orbit. But it is hard to see any clear conceptual reason to expect a positive answer in general.

\section{SUPPORT VARIETIES AND RANK VARIETIES}

Premet's proof of the Kac-Weisfeiler conjecture implicitly involves the notion of support variety of a module. He has shortened his original proof somewhat by developing this theme more fully in [64], [65]. We digress briefly to summarize some of the ideas involved, referring the reader especially to the work of FriedlanderParshall [14], [15] and Jantzen [30], [31], [32].

As in the modular representation theory of finite groups, study of a suitable (finitely-generated) cohomology ring and its action on the Ext-groups of a module leads to the introduction of an affine variety which encapsulates information about the "complexity" of the module, measured in terms of its minimal projective resolutions. In the Lie algebra setting this is based on the restricted cohomology theory introduced by Hochschild [22]. (See Feldvoss [13] and Nakano [53] for surveys of recent work.)

The combined work of Friedlander-Parshall and Jantzen leads to a more downto-earth formulation. Define $\mathcal{N}_{p}(\mathfrak{g})$ to be the set of all $x \in \mathfrak{g}$ satisfying $x^{[p]}=0$. This is a conical subvariety of the nilpotent variety $\mathcal{N}$ (and equal to it if $p$ is at least the Coxeter number). Now take an arbitrary $U_{\chi}(\mathfrak{g})$-module $M$ and restrict it to the subalgebra $\langle x\rangle$ of $U_{\chi}(\mathfrak{g})$ generated by $0 \neq x \in \mathcal{N}_{p}(\mathfrak{g})$ (which is $p$-dimensional and has a unique simple module). The rank variety of $M$ is defined to be the set of those $x$ for which the restriction of $M$ to $\langle x\rangle$ is not projective, together with 0 . It is again a conical variety, equal to 0 just when $M$ is a projective $U_{\chi}(\mathfrak{g})$-module. (The cohomological support variety maps bijectively to the rank variety, and therefore behaves similarly.)

Premet [65] has obtained a precise upper bound for the rank varieties of $U_{\chi}(\mathfrak{g})$ modules. Define the "centralizer" of $\chi$ to be $\mathfrak{z}_{\mathfrak{g}}(\chi):=\{z \in \mathfrak{g} \mid \chi([z, \mathfrak{g}])=0\}$. 
Theorem. The rank variety of every $U_{\chi}(\mathfrak{g})$-module lies in $\mathcal{N}_{p}(\mathfrak{g}) \cap \mathfrak{z}_{\mathfrak{g}}(\chi)$. Moreover, there is a module having precisely this variety.

\section{INDUCED MODULES AND SIMPLE MODULES}

In this kind of representation theory one does not usually expect to be able to construct all of the simple modules explicitly. (There are, however, some special constructions in the literature: see for example Peters [58], Peters-Shi [59].) Instead, the usual strategy is to construct a sufficiently large family of "standard" modules so that all simple modules appear naturally as composition factors of these. Ideally, each standard module would have a unique simple quotient or unique simple submodule.

In the case of $U_{\chi}(\mathfrak{g})$ it is easy to get started. Without loss of generality, we may fix a Borel subalgebra $\mathfrak{b}=\mathfrak{h} \oplus \mathfrak{n}^{+}$of $\mathfrak{g}$ so that $\chi$ vanishes on the nilradical $\mathfrak{n}^{+}$. Each $\lambda \in \mathfrak{h}^{*}$ affords a 1-dimensional representation $K_{\lambda}$ of $\mathfrak{h}$, which lifts to a representation of $\mathfrak{b}$ by making $\mathfrak{n}^{+}$act as 0 . This defines a $U_{\chi}(\mathfrak{b})$-module only for the $p^{r}$ possible $\lambda \in \mathfrak{h}^{*}$ which satisfy for all simple roots $\alpha$ :

$$
\lambda\left(h_{\alpha}^{p}\right)-\lambda\left(h_{\alpha}\right)=\chi\left(h_{\alpha}\right)^{p} .
$$

In this case we can set $Z_{\chi}(\lambda):=U_{\chi}(\mathfrak{g}) \otimes_{U_{\chi}(\mathfrak{b})} K_{\lambda}$. (Just write $Z(\lambda)$ if $\chi=0$.) The vector $v:=1 \otimes 1$ has weight $\lambda$, and we get all of $Z_{\chi}(\lambda)$ by applying various negative root vectors $f_{\alpha}$ to $v$. This is an induced module construction analogous to the construction of Verma modules in characteristic 0 . So $Z_{\chi}(\lambda)$ is sometimes called a "baby Verma module".

Theorem. All modules $Z_{\chi}(\lambda)$ have dimension $p^{N}$, where $N$ is the number of positive roots. Every simple $U_{\chi}(\mathfrak{g})$-module is a quotient of some $Z_{\chi}(\lambda)$ with $\lambda$ as above.

For the proof see Rudakov [68] (cf. [17, Prop. 1.5]). When $\chi \neq 0$, it often happens that $Z_{\chi}(\lambda) \cong Z_{\chi}(\mu)$ even when $\lambda \neq \mu$ in $X / p X$, as we will soon see. So it is tricky to parametrize simple modules by weights and decide how many there are. In the restricted case, $Z(\lambda)$ has a unique simple quotient, which can unambiguously be labelled $L(\lambda)$. But examples found by Jantzen show that this fails for some choices of $\mathfrak{b}$ when $\chi \neq 0$; indeed, in extreme cases $Z_{\chi}(\lambda)$ can even be decomposable. How $\mathfrak{b}$ is chosen relative to $\chi$ thus becomes an important issue. (Even when $\chi=0$, variation of $\mathfrak{b}$ affects the structure of induced modules in ways not yet fully understood: see [25].)

In spite of these obstacles, we expect at least the following to be true (perhaps with mild restrictions on $p$ ):

Conjecture. For arbitrary $\chi \in \mathfrak{g}^{*}$, there are at most $p^{r}$ non-isomorphic simple $U_{\chi}(\mathfrak{g})$-modules.

\section{Central characters}

While it is easy to construct induced modules, it is not so easy to determine their composition factors. One useful tool is the action of the center $Z(\mathfrak{g})$ of $U(\mathfrak{g})$ (or its canonical image in $\left.U_{\chi}(\mathfrak{g})\right)$. In characteristic 0 one gets a precise description of the center using the Harish-Chandra homomorphism: it is isomorphic to the algebra of Weyl group invariants in the universal enveloping algebra of a Cartan subalgebra. Moreover, the center acts on Verma modules by scalars, and the resulting central characters are easily computed in terms of highest weights. 
Things get more complicated in characteristic $p$, where $Z:=Z(\mathfrak{g})$ has a large subalgebra $\mathcal{O}$ generated by the elements $x^{p}-x^{[p]}$. But the subalgebra $Z^{G}$ of $G$ invariants in $Z$ turns out to behave much like the full center in characteristic 0 (see Veldkamp [78] and Kac-Weisfeiler [39], where some restrictions are imposed on $p$ and the type of $\mathfrak{g})$. In particular, $Z^{G}$ acts on the induced modules $Z_{\chi}(\lambda)$ by analogous central characters: each $z \in Z^{G}$ acts on $Z_{\chi}(\lambda)$ by a scalar $\theta_{\lambda}(z)$, thus defining an algebra homomorphism $\theta_{\lambda}: Z^{G} \rightarrow K$.

It is then crucial to determine when two characters coincide. In characteristic 0 , Harish-Chandra's approach led to an elegant formulation: when $\lambda, \mu$ are integral weights, central characters labelled by $\lambda$ and $\mu$ coincide if and only if there exists $w \in W$ for which $\mu=w \cdot \lambda:=w(\lambda+\rho)-\rho$, with $\rho$ the sum of fundamental weights (= half-sum of positive roots). When $\lambda, \mu$ fail to be integral, only a subgroup of $W$ is involved here. The characteristic $p$ analogue was worked out by Kac-Weisfeiler [39, Thm. 2] under some restrictions on $p$ and the type of $\mathfrak{g}$ (see also [24] for the restricted case). We give just a rough formulation:

Theorem. Assume $\lambda, \mu \in \mathfrak{h}^{*}$ are differentials of weights in $X$. Under mild restrictions on $p, \theta_{\lambda}=\theta_{\mu}$ if and only if $\mu=w \cdot \lambda$ for some $w$ in $W$.

We say in this situation that $\lambda, \mu$ are $W$-linked. This amounts to saying that corresponding weights $\lambda$ and $\mu$ in $X$ are conjugate under the action of the extended affine Weyl group relative to $p$ : the semidirect product of $W$ with the translation group $p X$. The affine Weyl group relative to $p$ is the subgroup involving just translations by $p$ times roots and is a Coxeter group to which Kazhdan-Lusztig theory applies.

The theorem shows that $Z_{\chi}(\lambda)$ and $Z_{\chi}(\mu)$ can share a composition factor only if $\lambda$ and $\mu$ are $W$-linked. If $\chi(\mathfrak{b})=0$ (the "nilpotent" case emphasized below), something more precise can be said: see Jantzen [35, Prop. 1.5], which extends the argument in [24] for $\chi=0$.

Theorem. Assume $\chi(\mathfrak{b})=0$. If weights $\lambda, \mu \in X / p X$ are $W$-linked, then $Z_{\chi}(\lambda)$ and $Z_{\chi}(\mu)$ determine the same element of the Grothendieck group of $U_{\chi}(\mathfrak{g})$-modules: they have the same composition factors, counting multiplicity.

This is proved by defining suitable intertwining maps and then comparing their kernels and cokernels.

\section{JoRDAN DECOMPOSITION IN $\mathfrak{g}^{*}$}

How can one get more insight into the induced modules $Z_{\chi}(\lambda)$ ? This depends in part on a more systematic study of the coadjoint orbits. In their 1976 paper [39], Kac and Weisfeiler showed how to carry over to $\mathfrak{g}^{*}$ the Jordan-Chevalley decomposition long used in the study of $G$ and $\mathfrak{g}$. Recall that in $\mathfrak{g}$ one has an intrinsic decomposition $x=x_{s}+x_{n}$ with $x_{s}$ semisimple, $x_{n}$ nilpotent, and $\left[x_{s}, x_{n}\right]=0$. In the setting of a restricted Lie algebra, an element is "semisimple" if it lies in the linear span of its images under the iterated $p$-map, while it is "nilpotent" if some power of the $p$-map kills it. The image of such an element in a restricted representation (such as the adjoint representation) is a semisimple or nilpotent linear operator in the usual sense.

Relative to a triangular decomposition $\mathfrak{g}=\mathfrak{n}^{-} \oplus \mathfrak{h} \oplus \mathfrak{n}^{+}$, semisimple elements are those with a $G$-conjugate in $\mathfrak{h}$, while nilpotent elements are those with a $G$ conjugate in $\mathfrak{n}^{+}\left(\right.$or $\left.\mathfrak{n}^{-}\right)$. 
When the Killing form of $\mathfrak{g}$ is nondegenerate, one can transfer the Jordan decomposition to $\mathfrak{g}^{*}$. In other cases, Kac and Weisfeiler were able to define such a decomposition intrinsically so as to satisfy the correct formal conditions. Using this technique, they could largely reduce the study of irreducible representations of $\mathfrak{g}$ to the study of $U_{\chi}(\mathfrak{g})$-modules when $\chi$ is nilpotent (as explained below).

\section{EXAMPLE: $\mathfrak{s l}(2, K)$}

The 3-dimensional Lie algebra $\mathfrak{g}=\mathfrak{s l}(2, K)$ suggests what we may expect to find in general. We leave aside the case $p=2$, which requires extra care, so $\mathfrak{g}$ is simple and has nondegenerate Killing form. We have a standard basis $\{h, e, f\}$, with brackets $[h e]=2 e,[h f]=-2 f,[e f]=h$.

Here there are three essentially different kinds of coadjoint orbits in $\mathfrak{g}^{*}$, represented in $\mathfrak{g}$ by $0, e$ (regular nilpotent), and the various nonzero multiples of $h$ (regular semisimple).

In the first case, $U_{\chi}(\mathfrak{g})=U^{[p]}(\mathfrak{g})$ is the restricted enveloping algebra, whose simple modules are of dimensions $1, \ldots, p$ and may be obtained by reduction mod $p$ from the classical highest weight modules of these dimensions for $\mathfrak{s l}(2, \mathbb{C})$. They may be labelled with "highest weights" $0,1, \ldots, p-1$ : the eigenvalue of $h$ on a vector killed by $e$. The induced modules $Z(\lambda)$ are of dimension $p$ and typically have two composition factors (labelled by linked weights $\lambda$ and $p-2-\lambda$ and having respective dimensions $\lambda+1, p-1-\lambda$ ). But one of them is already simple (and projective) of dimension $p$ : the Steinberg module, of highest weight $p-1$. Other projective covers have dimension $2 p$ and can be filtered by two induced modules.

In the (regular) nilpotent case, all of the induced $U_{\chi}(\mathfrak{g})$-modules $Z_{\chi}(\lambda)$ turn out to be simple (of dimension $p$ ). They can again be parametrized by the weights $0,1, \ldots, p-1$, but here each linked pair $\{\lambda, p-2-\lambda\}$ determines a single module. Its projective cover is an extension of the simple module by itself. The one exception is the Steinberg-type module of weight $p-1$, which is both simple and projective.

In the (regular) semisimple cases, $U_{\chi}(\mathfrak{g})$ is semisimple. The induced modules $Z_{\chi}(\lambda)$ are all simple (as in the regular nilpotent case) and are uniquely parametrized by weights (as in the restricted case). But here the weight parameters no longer lie in the prime field of $K$. Instead, they are roots of the separable polynomial $\lambda^{p}-\lambda-\chi(h)^{p}$, reflecting the fact that $h^{p}-h$ must act on a simple module by the scalar $\chi(h)^{p}$.

For details see Friedlander-Parshall [17, §2]. (Further aspects are treated by Benkart-Osborn [3], Pollack [60], Premet [61].)

Rudakov and Shafarevich [73] used the early work of Zassenhaus to organize all of the irreducible representations into a single geometric picture when $\mathfrak{g}=\mathfrak{s l}(2, K)$ and $p \neq 2$ : the Zassenhaus variety, whose simple points correspond to representations of dimension $p$. It would be very interesting to work out a similar description for higher ranks, but so far only a bare outline exists.

\section{The SEMisimple CASE}

The "semisimple" case lives up to its name. The following theorem goes back to Rudakov [68] and Kac-Weisfeiler [39]. The ideas were later reworked and extended by Friedlander-Parshall [17, §3].

Theorem. Assume $p$ is good for $G$. 
1. If $\chi$ is semisimple, there are $p^{r}$ isomorphism classes of simple $U_{\chi}(\mathfrak{g})$-modules, where $r=\operatorname{dim} \mathfrak{h}$. Each has a unique $\mathfrak{b}$-stable line of weight $\lambda$, and the $p^{r}$ distinct choices for $\lambda$ are those satisfying $\lambda\left(h_{\alpha}\right)^{p}-\lambda\left(h_{\alpha}\right)-\chi\left(h_{\alpha}\right)^{p}=0$ for all simple roots $\alpha$.

2. The algebra $U_{\chi}(\mathfrak{g})$ is semisimple if and only if $\chi$ is regular semisimple.

\section{Reduction to the nilpotent CASE}

When $\chi$ is no longer semisimple, the representation theory of $U_{\chi}(\mathfrak{g})$ can be reduced to that of a lower rank Lie algebra. This was also worked out by KacWeisfeiler and reformulated by Friedlander-Parshall.

Say $\chi$ corresponds to $x \in \mathfrak{g}$ (if the Killing form is nondegenerate). Given the Jordan decomposition $x=x_{s}+x_{n}$ of $x \in \mathfrak{g}$ (with $x_{n} \neq 0$ ), the key point is that $x_{n}$ lies in the centralizer $\mathfrak{l}$ of $x_{s}$, a reductive subalgebra of $\mathfrak{g}$ whose semisimple derived algebra $\mathfrak{l}^{\prime}$ has smaller rank than $\mathfrak{g}$. The theory for simple Lie algebras generalizes readily to the semisimple case. Now one can inductively reduce matters to the study of a nilpotent orbit: $U_{\chi}(\mathfrak{g})$ is Morita equivalent to the tensor product of a commutative semisimple algebra with $U_{\psi}\left(\mathfrak{l}^{\prime}\right)$, where $\psi$ lies in a nilpotent coadjoint orbit corresponding to $x_{n}$.

So Jordan decomposition allows us to focus on the essential case, where $\chi$ is nilpotent.

\section{The NiLPotent CASE}

From now on $\chi$ always denotes a nilpotent element of $\mathfrak{g}^{*}$.

With a few exceptions when $p$ is bad, the orbit structure of the nilpotent variety $\mathcal{N}$ of $\mathfrak{g}$ resembles closely that of the nilpotent variety of a simple Lie algebra over $\mathbb{C}$. There are only finitely many $G$-orbits in $\mathcal{N}$, which have been enumerated and classified in analogy with the classical Dynkin-Kostant theory, adapted by BalaCarter and Pommerening to good prime characteristic. The Jordan normal form for $\mathfrak{s l}(n, K)$ is of course a prototype of this classification. But in general things get much more complicated.

What does it mean for $\chi$ to be nilpotent? For a fixed Borel subalgebra $\mathfrak{b}=\mathfrak{h} \oplus \mathfrak{n}^{+}$, every nilpotent element of $\mathfrak{g}$ has a conjugate in $\mathfrak{n}^{+}$. When the Killing form is nondegenerate, the orthogonal complement of $\mathfrak{n}^{+}$is precisely $\mathfrak{b}$. Saying that $\chi$ is nilpotent then translates into the condition that $\chi$ vanishes on a $G$-conjugate of $\mathfrak{b}$. If $\chi(\mathfrak{b})=0$, then $\chi\left(h_{\alpha}\right)=0$ for all roots $\alpha$. This means that for any $U_{\chi}(\mathfrak{b})$-module $M, h_{\alpha}^{[p]}-h_{\alpha}$ acts by the scalar 0 . In turn, for any weight $\lambda$ of $\mathfrak{h}$ on $M, \lambda\left(h_{\alpha}\right)$ lies in the prime field $\mathbb{F}_{p}$.

Thus, when $\chi$ is nilpotent and $\mathfrak{b}$ is chosen so that $\chi(\mathfrak{b})=0$, the weights of any $U_{\chi}(\mathfrak{g})$-module are integral, i.e., reductions modulo $p$ of weights in $X$. So induced modules $Z_{\chi}(\lambda)$ are parametrized by elements of $X / p X$. The only catch is that (unlike the case $\chi=0$ ) numerous weights $\lambda$ may parametrize the same module. But these all lie in one $W$-linkage class.

\section{STANDARD LEVI FORM}

Fix a Borel subalgebra $\mathfrak{b}=\mathfrak{h} \oplus \mathfrak{n}^{+}$, hence a system of simple roots, and assume $\chi(\mathfrak{b})=0$. Following Friedlander-Parshall $[18, \S 3]$, we say that $\chi$ has standard Levi form if there is a set $I$ of simple roots such that $\chi\left(f_{\alpha}\right)=1$ for all $\alpha \in I$ while $\chi\left(f_{\alpha}\right)=0$ for all other positive roots $\alpha$. If $\chi$ corresponds (under the Killing 
form duality) to an element $x \in \mathfrak{n}^{+}$, it is equivalent to say that $x$ is the sum of root vectors $e_{\alpha}(\alpha \in I)$ : a typical regular nilpotent element of a Levi factor in the parabolic subalgebra determined by $I$. An extreme example is $\chi=0$.

The techniques developed by Friedlander-Parshall and by Jantzen are mainly effective in the case of standard Levi form. In particular, this is the only case in which we currently have a precise classification of simple $U_{\chi}(\mathfrak{g})$-modules by "highest weights". When $\mathfrak{g}=\mathfrak{s l}(n, K)$, all nilpotent $\chi \in \mathfrak{g}^{*}$ are easily seen to be conjugate to those having standard Levi form. But for other simple Lie algebras there are "extra" nilpotent $\chi$, such as those corresponding to subregular nilpotent elements when $\mathfrak{g}$ is the exceptional 14-dimensional algebra of type $G_{2}$.

Theorem. Assume $\chi$ has standard Levi form relative to the Borel subalgebra $\mathfrak{b}$.

1. Each $Z_{\chi}(\lambda)$ has a unique simple quotient, which may be denoted $L_{\chi}(\lambda)$. In particular, $Z_{\chi}(\lambda)$ is indecomposable.

2. The simple modules $L_{\chi}(\lambda)$ and $L_{\chi}(\mu)$ are isomorphic if and only if $\lambda$ and $\mu$ are $W_{I}$-linked in $X / p X$, where $W_{I}$ is the subgroup of $W$ generated by reflections relative to all $\alpha \in I$.

See Friedlander-Parshall [18, Prop. 2.3, Cor. 3.5], where some restrictions are imposed on $p$, and Jantzen $[35,1.6]$. In the case of $\mathfrak{s l}(n, K)$, this parametrization of simple modules with nilpotent $p$-characters was first worked out by Panov [56] (and recovered in [18]).

How does standard Levi form come into play in the second statement of the theorem? Suppose $\alpha \in I$. By applying $f_{\alpha}, f_{\alpha}^{2}, \ldots$ to a primitive vector $v$ in $Z_{\chi}(\lambda)$, we get a string of weight vectors of respective weight $\lambda-\alpha, \lambda-2 \alpha, \ldots$ For some $k \leq p$, standard commutation formulas in $\mathfrak{g}$ show that we arrive at a new primitive vector $v^{\prime}=f_{\alpha}^{k} v$ of weight $\lambda-k \alpha=s_{\alpha} \cdot \lambda$ ( $s_{\alpha}$ being the reflection in $W$ relative to $\alpha$ ). But $f_{\alpha}^{p}$ acts as the identity operator. This implies that $v=f_{\alpha}^{p} v=f_{\alpha}^{p-k} v^{\prime}$. Thus $v^{\prime}$ can equally be taken as a generator of $Z_{\chi}(\lambda)$. The first statement of the theorem then shows that $L_{\chi}(\lambda) \cong L_{\chi}\left(s_{\alpha} \cdot \lambda\right)$. Now iterate.

\section{BLOCKS}

One easy consequence of the preceding theorem is an explicit determination of the blocks (indecomposable two-sided ideals) of $U_{\chi}(\mathfrak{g})$ when $\chi$ has standard Levi form. This generalizes the case $\chi=0$ treated in [24].

Theorem. If $\chi$ has standard Levi form, the blocks of $U_{\chi}(\mathfrak{g})$ are in natural bijection with the $W$-linkage classes of weights in $X / p X$.

To explain this, recall from the general theory of finite-dimensional algebras that each simple module occurs as a composition factor of precisely one block, and that all composition factors of an indecomposable module must belong to a single block. When $\chi$ has standard Levi form (relative to $\mathfrak{b}$ ), we know that all $Z_{\chi}(\lambda)$ are indecomposable and that their unique simple quotients $L_{\chi}(\lambda)$ exhaust the simple $U_{\chi}(\mathfrak{g})$-modules. If $L_{\chi}(\lambda)$ and $L_{\chi}(\mu)$ occur in the same block, their central characters (§11) must coincide and thus $\lambda$ and $\mu$ must be $W$-linked. In the reverse direction, the second theorem in $\S 11$ shows - without assuming standard Levi form - that $Z_{\chi}(\lambda)$ involves composition factors of all possible weights $W$-linked to $\lambda$.

We expect this result to hold in general: 
Conjecture. If $\chi$ is nilpotent, the blocks of $U_{\chi}(\mathfrak{g})$ are in natural bijection with $W$ linkage classes of weights in $X / p X$. In particular, all of these finite-dimensional algebras have the same number of blocks.

To prove this it would be enough to show that there always exists a Borel subalgebra yielding induced modules $Z_{\chi}(\lambda)$ which are indecomposable - for example, by virtue of having unique simple quotients. The first open case is that of the subregular nilpotent orbit in type $G_{2}$ mentioned above.

It is tempting to conjecture that for arbitrary $\chi \in \mathfrak{g}^{*}$, the number of nonisomorphic simple modules in each block should be no greater than $|W|$. For "generic" blocks we might also ask whether this number of simple modules always divides $|W|$. This is true when $\chi$ is nilpotent and has standard Levi type, since then the number is $\left|W / W_{I}\right|$ (see $\S 17$ ). Here the generic blocks involve $p$-regular weights: those for which the $W$-linkage class has size $|W|$.

\section{The Regular niLPotent orbit}

At the opposite extreme to the zero orbit in $\mathfrak{g}$ is the unique open orbit, consisting of regular nilpotent elements - those whose centralizers have smallest possible dimension $r$ and whose orbit therefore has dimension $2 N$. In terms of our Chevalley basis of $\mathfrak{g}$, we can take as orbit representative the sum of all $e_{\alpha}$ ( $\alpha$ simple). Thus the corresponding character $\chi \in \mathfrak{g}^{*}$ has standard Levi form.

Premet's Theorem says that the dimensions of all $U_{\chi}(\mathfrak{g})$-modules are divisible by $p^{N}$. This special case can in fact be seen fairly directly. Under mild restrictions on $p$, Friedlander-Parshall [17, Theorem 4.3] show:

Theorem. Let $\chi$ be regular nilpotent. For all weights $\lambda$, we have $Z_{\chi}(\lambda)=L_{\chi}(\lambda)$. Moreover, all weights in a given linkage class determine a single simple $U_{\chi}(\mathfrak{g})$ module. In particular, each block of $U_{\chi}(\mathfrak{g})$ involves just one simple module $L_{\chi}(\lambda)$. Its projective cover $P_{\chi}(\lambda)$ involves $|W \cdot \lambda|$ copies of $L_{\chi}(\lambda)$.

The assertion about the dimension of $P_{\chi}(\lambda)$ involves an overall dimension comparison: as in the case of any finite-dimensional algebra over $K, \operatorname{dim} U_{\chi}(\mathfrak{g})$ is obtained by multiplying the dimension of each simple module by the dimension of its projective cover, then summing. But the internal structure of $P_{\chi}(\lambda)$ is more difficult to describe. All one knows from the fact that $U_{\chi}(\mathfrak{g})$ is a "symmetric" algebra is that $P_{\chi}(\lambda)$ has $L_{\chi}(\lambda)$ as both head and socle. When the $W$-linkage class of $\lambda$ has the generic size $|W|$, Jantzen $[37,10.12]$ points out (in the spirit of arguments in [2]) that the $|W|$-dimensional endomorphism algebra of $P_{\chi}(\lambda)$ is isomorphic to the "coinvariant" algebra of $W$. This leads to a more precise picture of the radical series, whose length is $N+1$. (Premet has announced more general results for arbitrary $\lambda$.)

\section{Calculations by Jantzen}

Until very recently there were few specific calculations involving non-restricted representations, apart from the regular nilpotent case and the Lie algebras $\mathfrak{s l}(2, K)$ and $\mathfrak{s l}(3, K)$. But by pushing Premet's methods further, Jantzen [35], [36] has managed to get explicit dimension formulas for simple modules in a number of further cases: the subregular nilpotent orbit in types $A_{n}$ and $B_{n}$ as well as the minimal (nonzero) nilpotent orbit in type $B_{2}$. The latter case involves intricate calculations, but at the same time provides strong evidence for a connection with 
Lusztig's recent work on affine Hecke algebras (described below). Along the way he calculates related Ext groups and Loewy series.

The subregular orbit in $\mathfrak{g}$ involves elements in standard Levi form only when $\mathfrak{g}$ is of type $A_{n}$ or $B_{n}$. Here Jantzen chooses a suitable nilpotent element and Borel subalgebra so that the resulting induced modules $Z_{\chi}(\lambda)$ are uniserial, with (generically) distinct composition factors. Thanks to standard Levi form, the simple modules are easily parametrized by families of weights. By studying maps among various induced modules, some of which are induced from larger parabolic subalgebras, he is eventually able to pin down all dimensions of simple modules for $U_{\chi}(\mathfrak{g})$. In keeping with Premet's Theorem, these are of the form $a p^{N-1}$, since the subregular orbit has codimension 2 in the $2 N$-dimensional nilpotent variety.

When $\mathfrak{g}=\mathfrak{s l}(3, K)$, he recovers the calculations of Kac [38] (described in [18, Ex. 3.6]). Here there are just three nilpotent orbits, corresponding to Jordan forms: 0 , the subregular orbit, and the regular orbit.

For the Lie algebra $\mathfrak{s o}(5, K)$ of type $B_{2}$, Jantzen works with an element of the (4-dimensional) minimal nilpotent orbit and a suitable Borel subalgebra. Here the results are more complicated, because the modules $Z_{\chi}(\lambda)$ generically have six composition factors, involving the four simple modules in a block with multiplicities 1 or 2 . If weights are parametrized by pairs $(r, s)$ (after a shift by $\rho$ ) with $r, s>0$ and $2 r+s<p$, the four typical dimensions are:

$$
\frac{s(p+2 r+s)}{2} p^{2}, r p^{3}, \frac{s(p-2 r-s)}{2} p^{2}, \frac{(2 p-s)(p-2 r-s)}{2} p^{2} .
$$

Note that by adding these dimensions, with the second and third counted twice, one obtains $p^{4}=\operatorname{dim} Z_{\chi}(\lambda)$. In this generic case, $Z_{\chi}(\lambda)$ has Loewy length 4 , with explicitly computed layers.

To keep track of weights, Jantzen exploits a natural action of a subtorus of $G$ which fixes $\chi$, or a related grading of the category of $U_{\chi}(\mathfrak{g})$-modules. This viewpoint is compatible with Lusztig's work on the affine Weyl group, which we now describe briefly.

\section{LUSZTIG'S PERIODIC $W$-GRAPHS}

Recently Lusztig $[49,13.17]$ has suggested, as a byproduct of a new construction of representations of affine Hecke algebras, how to compute the composition factor multiplicities of the modules $Z_{\chi}(\lambda)$ (for "generic" $\lambda$ ) when $\chi$ has standard Levi form. More precisely, he fixes a nonempty set $I$ of simple roots and works in a correlated "strip" of alcoves for the affine Weyl group along one or more walls of the fundamental Weyl chamber, attaching polynomials in $v^{-1}$ to the alcoves. When evaluated at $v=1$, these should provide the desired multiplicities (in an inverse sense). His calculations for types $A_{2}$ and $B_{2}$ agree with Jantzen's results. He also calculates the polynomials relevant to the 6-dimensional and 8-dimensional nilpotent orbits of $G_{2}$, for which no explicit representation-theoretic results are yet known.

It is noteworthy that this setup incorporates already the kind of grading (or torus action) which plays an essential part in Jantzen's bookkeeping. All of this extends in a promising way Lusztig's earlier conjectures for $\chi=0$ (see [46]). Even so, the composition factor multiplicities alone are not enough to predict the dimensions of the simple modules. 
One can hope that the polynomials themselves will give information about the Loewy series of $Z_{\chi}(\lambda)$. Lusztig's upper bound on degrees of the polynomials would then suggest a generic Loewy length for $Z_{\chi}(\lambda): 1$ plus the number of positive roots outside $I$. This is consistent with the earlier estimate $N+1$ when $\chi=0$ and with Jantzen's rank 2 calculations.

Many questions remain. For example, can one relate Lusztig's constructions for varying choices of $I$ ? And is there any way to extend his ideas to cover the cases when $\chi$ does not have standard Levi form? Above all, it remains to discover an intrinsic connection between the Hecke algebra formalism and the modular representation theory of Lie algebras.

\section{Deformations}

It has long been hoped that a close study of the non-restricted representations of $\mathfrak{g}$ would lead indirectly, by a "deformation" process, to an understanding of restricted representations. This theme is developed in the series of papers by Friedlander and Parshall, where the "Steinberg modules" associated to the weight $(p-1) \rho$ for the various nilpotent $\chi$ are seen to form a deformation family [18, §4].

There is a natural partial ordering of nilpotent orbits in $\mathfrak{g}$ (or in $\mathfrak{g}^{*}$ ), with two orbits related if one lies in the closure of the other. In types $A_{2}$ and $B_{2}$ (where the ordering happens to be linear), there are respectively 3 and 4 nilpotent orbits. The representation theory of $U_{\chi}(\mathfrak{g})$ is relatively uncomplicated when $\chi$ is regular, but becomes quite intricate by the time we reach the 0 orbit. A step-by-step deformation through the orbits for $A_{2}$ and $B_{2}$ has been worked out explicitly by Jantzen. The idea is to introduce an extra parameter $t$, allowing one to pass from an orbit into its closure by setting $t=0$. For example, in $\mathfrak{s l}(3, K)$, we can start with regular nilpotent elements of the form $e_{\alpha}+t e_{\beta}(\alpha, \beta$ simple roots, $t \neq 0)$ and then pass to the subregular orbit when $t=0$.

The details for types $A_{2}$ and $B_{2}$ are already somewhat complicated, and still impossible to predict a priori. But the patterns exhibited are essentially independent of $p$, lending support to the hope that they may depend just on the root system and Weyl group (or affine Weyl group).

\section{Relations With The FlaG VARIETy}

As we indicated earlier, the relationship between representations of $G$ and representations of $\mathfrak{g}$ is more subtle in characteristic $p$ than in characteristic 0 . One actually has to compare two families of "standard" modules: the algebraically constructed induced $U^{[p]}(\mathfrak{g})$-modules $Z(\lambda), \lambda \in X / p X$, and the geometrically constructed induced $G$-modules $H^{0}(\lambda), \lambda \in X^{+}$("dual Weyl modules"). The latter arise as global sections of line bundles on the flag variety $\mathcal{B}$, parametrized by dominant weights. The modules $Z(\lambda)$ all have dimension $p^{N}$, whereas the modules of global sections have dimensions given by Weyl's classical formula, starting with the 1-dimensional trivial module $H^{0}(0)$ for $G$.

Even though the modules $H^{0}(\lambda)$ are not defined intrinsically in terms of $\mathfrak{g}$, Jantzen's work on "generic decomposition patterns" shows that they interact closely with the $Z(\lambda)$. And Lusztig's older conjecture on characters and dimensions is formulated explicitly for $G$-modules rather than for $\mathfrak{g}$-modules.

When $\chi$ is nilpotent but nonzero, it is tempting to look for a similar family of geometrically defined "standard" modules which would interact in a parallel way 
with the $Z_{\chi}(\lambda)$. There is a natural candidate for the geometric setting: the fixed point variety $\mathcal{B}_{x}$ of a nilpotent element $x$ corresponding to $\chi$. This is a single point when $x$ is regular and is all of $\mathcal{B}$ when $x=0$. If we regard $\mathcal{B}$ as the collection of all Borel subalgebras of $\mathfrak{g}$, then $\mathcal{B}_{x}$ comprises those which contain $x$. These fixed point varieties are the Springer fibres in a desingularization of the nilpotent variety (cf. $[26$, Chapter 6]).

While $\mathfrak{g}$ need not act on global sections of line bundles restricted to $\mathcal{B}_{x}$, recent work of Mirković and Rumynin [52] shows how to obtain $U_{\chi}(\mathfrak{g})$-modules by working with global sections of vector bundles on an expanded "Frobenius neighborhood". The hope is that this construction will yield directly some of the simple $U_{\chi}(\mathfrak{g})$ modules (including those of least possible dimension) and will suggest a link with Kazhdan-Lusztig theory. But many technical problems still need to be resolved.

Their approach also promises to relate the $Z_{\chi}(\lambda)$ systematically to their geometrically constructed "standard modules". This may help to explain the variation in structure of the $Z_{\chi}(\lambda)$ as one varies the choice of Borel subalgebra containing a fixed nilpotent element (corresponding to $\chi$ ). For example, it is suspected that choosing $\mathfrak{b}$ to lie on only one irreducible component of $\mathcal{B}_{x}$ will yield modules $Z_{\chi}(\lambda)$ with unique simple quotients. On the other hand, Jantzen's examples for $\mathfrak{s l}(3, K)$ show already that the choice of a Borel subalgebra lying in more than one component can lead to a more complicated head or socle.

\section{Quantum groups at a Root of Unity}

Almost everything we have been discussing here has a precise parallel in the case of quantum groups - by which we mean quantum enveloping algebras in the sense of Drinfeld and Jimbo - when the quantum parameter is taken to be a root of unity. Here we just cite a few of the key references. A quantum analogue of the restricted enveloping algebra was constructed by Lusztig [47], [48], with further important developments by Andersen-Jantzen-Soergel [2]; see also Ginzburg-Kumar [20], Kazhdan-Verbitsky [40], Kumar [42]. For not necessarily restricted representations, a suitable framework was developed by DeConcini-Kac [8], DeConciniKac-Procesi [9], [10], [11]; see also Chari-Pressley [5].

The study of quantum groups has brought new methods as well as new motivation to the study of modular representations - a branch of representation theory which at times wanders far from the mainstream of traditional Lie theory, but remains deep.

\section{REFERENCES}

1. H.H. Andersen, The irreducible characters for semi-simple algebraic groups and for quantum groups, pp. 732-743, Proc. Intern. Congress Math. (Zürich, 1994), Birkhäuser, Basel, 1995. MR 97m:20051

2. H.H. Andersen, J.C. Jantzen, and W. Soergel, Representations of quantum groups at a p-th root of unity and of semisimple groups in characteristic p: independence of $p$, Astérisque 220 (1994). MR 95j:20036

3. G.M. Benkart and J.M. Osborn, Representations of rank one Lie algebras of characteristic p, pp. 1-37, Lie Algebras and Related Topics, Lecture Notes in Math., vol. 933, Springer-Verlag, Berlin, 1982. MR 84c:17004

4. A.J. Berkson, The u-algebra of a restricted Lie algebra is Frobenius, Proc. Amer. Math. Soc. 15 (1964), 14-15. MR 28:2132

5. V. Chari and A. Pressley, Representations of modular Lie algebras through quantum groups, C.R. Acad. Sci. Paris 317 (1993), 723-728. MR 94j:17020 
6. C.W. Curtis, Noncommutative extensions of Hilbert rings, Proc. Amer. Math. Soc. 4 (1953), 945-955. MR 15:498g

7. — Representations of Lie algebras of classical type with applications to linear groups, J. Math. Mech. 9 (1960), 307-326. MR 22:1634

8. C. DeConcini and V.G. Kac, Representations of quantum groups at roots of 1, pp. 471-506, Operator algebras, unitary representations, enveloping algebras, and invariant theory, Progr. Math., vol. 92, Birkhäuser, Boston, 1990. MR 92g:17012

9. C. DeConcini, V.G. Kac, and C. Procesi, Quantum coadjoint action, J. Amer. Math. Soc. 5 (1992), 151-189. MR 93f: 17020

10. Some remarkable degenerations of quantum groups, Comm. Math. Phys. 157 (1993), 405-427. MR 94i:17019

11. S_ Some quantum analogues of solvable Lie groups, pp. 41-65, Geometry and analysis (Bombay, 1992), Tata Inst. Fund. Res., Bombay, 1995. MR 96h:17015

12. R. Farnsteiner, Periodicity and representation type of modular Lie algebras, J. Reine Angew. Math. 464 (1995), 47-65. MR 97a:17019

13. J. Feldvoss, Homological topics in the representation theory of restricted Lie algebras, pp. 69 119, Lie algebras and their representations (Seoul, 1995), Contemp. Math., 194, Amer. Math. Soc., Providence, RI, 1996. MR 97e:17026

14. E.M. Friedlander and B.J. Parshall, Support varieties for restricted Lie algebras, Invent. Math. 86 (1986), 553-562. MR 88f: 17018

15. _ Geometry of p-unipotent Lie algebras, J. Algebra 109 (1987), 25-45. MR 89a:17017

16. , Rational actions associated to the adjoint representation, Ann. Sci. École Norm. Sup. (4) 20 (1987), 215-226. MR 88k:14026

17. $ـ$ Modular representation theory of Lie algebras, Amer. J. Math. 110 (1988), 10551094. MR 89j:17015

18. _ Deformations of Lie algebra representations, Amer. J. Math. 112 (1990), 375-395. MR 91e: 17012

19. - Induction, deformation, and specialization of Lie algebra representations, Math. Ann. 290 (1991), 473-489. MR 92h:17021

20. V. Ginzburg and S. Kumar, Cohomology of quantum groups at roots of unity, Duke Math. J. 69 (1993), 179-198. MR 94c: 17026

21. G. Hiss, Die adjungierten Darstellungen der Chevalley-Gruppen, Arch. Math. 42 (1984), 408-416. MR 85k:20134

22. G. Hochschild, Cohomology of restricted Lie algebras, Amer. J. Math. 76 (1954), 555-580. MR 16:019a

23. G.M.D. Hogeweij, Almost classical Lie algebras, Indag. Math. 44 (1982), I, 441-452; II, 453-460. MR 84f: 17007

24. J.E. Humphreys, Modular representations of classical Lie algebras and semisimple groups, J. Algebra 19 (1971), 51-79. MR 44:271

25. __ Induced modules for semisimple groups and Lie algebras, pp. 341-349, Lie Algebras and Related Topics (Conf. Proc. of Canad. Math. Soc., vol. 5), Amer. Math. Soc., Providence, RI, 1986. CMP 18:10

26. _ Conjugacy Classes in Semisimple Algebraic Groups, Math. Surveys Monographs, vol. 43, Amer. Math. Soc., Providence, RI, 1995. MR 97i:20057

27. _ Comparing modular representations of semisimple groups and their Lie algebras, pp. 69-80, Modular Interfaces: Modular Lie Algebras, Quantum Groups, and Lie Superalgebras, International Press, Cambridge, MA, 1997. CMP 98:05

28. N. Jacobson, Restricted Lie algebras of characteristic p, Trans. Amer. Math. Soc. 50 (1941), 15-25. MR 3:103g

29. _ Lie Algebras, Wiley Interscience, New York, 1962; Dover reprint, 1979. MR 80k:17001

30. J.C. Jantzen, Kohomologie von p-Lie-Algebren und nilpotente Elemente, Abh. Math. Sem. Univ. Hamburg 56 (1986), 191-219. MR 88e:17019

31. __ Restricted Lie algebra cohomology, pp. 91-108, Algebraic Groups (Utrecht, 1986), Lecture Notes in Math., vol. 1271, Springer-Verlag, Berlin, 1987. MR 89h:17023

32. _ Support varieties of Weyl modules, Bull. London Math. Soc. 19 (1987), 238-244. MR 88e: 17008

33. _ Representations of Algebraic Groups, Academic Press, Boston, 1987. MR 89c:20001 
34. Lectures on Quantum Groups, Grad. Studies in Math., vol. 6, Amer. Math. Soc., Providence, RI, 1996. MR 96m:17029

35. _ Subregular nilpotent representations of $\mathfrak{s l}_{n}$ and $\mathfrak{s o}_{2 n+1}$, Math. Proc. Cambridge Philos. Soc. (to appear).

36. __ Representations of $\mathfrak{s o}_{5}$ in prime characteristic, Aarhus Univ. Preprint Series, No. 13, 1997.

37. _ Representations of Lie algebras in prime characteristic, Proc. NATO Advanced Study Institute, Representation Theories and Algebraic Geometry, Montreal, 1997 (to appear).

38. V.G. Kac, Irreducible representations of Lie algebras of classical type [Russian], Uspekhi Mat. Nauk 27 (1972), no. 5, 237-238. MR 53:565

39. V. Kac and B. Weisfeiler, Coadjoint action of a semi-simple algebraic group and the center of the enveloping algebra in characteristic p, Indag. Math. 38 (1976), 136-151. MR 54:5364

40. D. Kazhdan and M. Verbitsky, Cohomology of restricted quantized universal enveloping algebras, pp. 107-115, Quantum Deformations of Algebras and Their Representations, Israel Math. Conf. Proc., vol. 7, Bar-Ilan University, 1993. MR 95b:17026

41. Ya. S. Krylyuk, The Zassenhaus variety of a classical semisimple Lie algebra in finite characteristic, Mat. Sb. (N.S.) 130 (1986), 475-487; English transl., Math. USSR-Sb. 58 (1987), 477-490. MR 88a:17023

42. S. Kumar, Representations of quantum groups at roots of unity, pp. 187-224, Quantum Topology, ed. D.N. Yetter, World Scientific, Singapore, 1994. MR 95m:17006

43. Z. Lin, Extensions between simple modules for Frobenius kernels, Math. Z. 207 (1991), 485499. MR 92g:20066

44. _ Loewy series of certain indecomposable modules for Frobenius subgroups, Trans. Amer. Math. Soc. 332 (1992), 391-409. MR 92j:20040

45. Z. Lin and D. Nakano, Algebraic group actions in the cohomology theory of Lie algebras of Cartan type, J. Algebra 179 (1996), 852-888. MR 96k:17011

46. G. Lusztig, Hecke algebras and Jantzen's generic decomposition patterns, Adv. Math. 37 (1980), 121-164. MR 82b:20059

47. __ Quantum groups at roots of 1, Geom. Dedicata 35 (1990), 89-114. MR 91j:17018

48. Finite dimensional Hopf algebras arising from quantized enveloping algebras, J. Amer. Math. Soc. 3 (1990), 257-296. MR 91e:17009

49. _ Periodic $W$-graphs, Represent. Theory 1 (1997), 207-279. CMP 97:16

50. A.A. Mil'ner, Irreducible representations of modular Lie algebras, Izv. Akad. Nauk SSSR Ser. Mat. 39 (1975), 1240-1259; English transl., Math. USSR-Izv. 9 (1975), 1169-1187. MR $\mathbf{5 7 : 9 7 7 4}$

51. __ Maximal degree of irreducible Lie algebra representations over a field of positive characteristic, Funktsional. Anal. i Prilozen. 14, no. 2, 67-68; English transl., Functional Anal. Appl. 14 (1980), 136-137. MR 81h:17012

52. I. Mirković and D. Rumynin, Geometric representation theory of restricted Lie algebras of classical type, in preparation.

53. D.K. Nakano, Complexity and support varieties for finite dimensional algebras, pp. 201-218, Lie algebras and their representations (Seoul, 1995), Contemp. Math., 194, Amer. Math. Soc., Providence, RI, 1996. MR 97c:17031

54. D.K. Nakano and R.D. Pollack, On the construction of indecomposable modules over restricted enveloping algebras, J. Pure Appl. Algebra 107 (1996), 61-73. MR 97e:17028

55. G.M. Nielsen, A determination of the minimal right ideals in the enveloping algebra of a Lie algebra of classical type, Ph.D. thesis, U. Wisconsin, 1963.

56. A.N. Panov, Irreducible representations of the Lie algebra sl $(n)$ over a field of positive characteristic, Mat. Sb. (N.S.) 128 (1985), 21-34; English transl., Math. USSR-Sb. 56 (1987), 19-32. MR 87h:17010

57. _ Irreducible representations of maximal dimension of a simple Lie algebra over a field of positive characteristic, Funktsional. Anal. i Prilozen. 23 (1989), no. 3, 80-81; English transl., Functional Anal. Appl. 23 (1989), 240-241. MR 90k:17038

58. K.M. Peters, Characters of modular torsion free representations of classical Lie algebras, Comm. Algebra 22 (1994), 4807-4826. MR 95h:17010

59. K.M. Peters and Z. Shi, A construction of modular representations of classical Lie algebras, Proc. Amer. Math. Soc. 122 (1994), 399-407. MR 95a:17010 
60. R.D. Pollack, Restricted Lie algebras of bounded type, Bull. Amer. Math. Soc. 74 (1968), 326-331. MR 36:2661

61. A.A. Premet, The Green ring of a simple three-dimensional Lie p-algebra [Russian], Soviet Math. (Iz. VUZ) 35 (1991), no. 10, 61-67. MR 93i:17007

62. __ An analogue of the Jacobson-Morozov theorem for Lie algebras of reductive groups of good characteristic, Trans. Amer. Math. Soc. 347 (1995), 2961-2988. MR 95k:17012

63. _ Irreducible representations of Lie algebras of reductive groups and the KacWeisfeiler conjecture, Invent. Math. 121 (1995), 79-117. MR 96g:17007

64. - Support varieties of non-restricted modules over Lie algebras of reductive groups, J. London Math. Soc. 55 (1997), 236-250; Corrigenda and addenda, J. London Math. Soc. (to appear). MR 98a:17026

65. _ Complexity of Lie algebra representations and nilpotent elements of the stabilizers of linear forms, Math. Z. (to appear).

66. A. Premet and S. Skryabin, Representations of restricted Lie algebras and equivariant families of associative algebras (to appear).

67. D. Quillen, On the endomorphism ring of a simple module over an enveloping algebra, Proc. Amer. Math. Soc. 21 (1969), 171-172. MR 39:252

68. A.N. Rudakov, On representations of classical semisimple Lie algebras of characteristic p, Izv. Akad. Nauk SSSR Ser. Mat. 34 (1970), 735-743; English transl., Math. USSR-Izv. 4 (1970), 741-749. MR 42:1872

69. — Weights of modular representations, Mat. Zametki 11 (1972), 397-402; English transl., Math. Notes 11 (1972), 244-247. MR 46:3583

70. _ Dimensions of certain irreducible representations of semi-simple Lie algebras of classical type over fields of finite characteristic [Russian], Trudy Sem. Petrovsk., No. 3 (1978), 147-160. MR 58:11046

71. _ Local universal algebras and reduced representations of Lie algebras, Izv. Akad. Nauk SSSR Ser. Mat. 43 (1979), 178-183; English transl., Math. USSR-Izv. 14 (1980), 169-174. MR 80d:17007

72. _ Reducible p-representations of a simple three-dimensional Lie p-algebra [Russian], Vestnik Moskov. Univ. Ser. I Mat. Mekh. 1982, no. 6, 45-49. MR 84g:17016

73. A.N. Rudakov and I.R. Shafarevich, Irreducible representations of a simple three-dimensional Lie algebra over a field of finite characteristic, Mat. Zametki 2 (1967), 439-454; English transl., Math. Notes 2 (1967), 760-767. MR 36:2662

74. J.R. Schue, Symmetry for the enveloping algebra of a restricted Lie algebra, Proc. Amer. Math. Soc. 16 (1965), 1123-1124. MR 32:2515

75. G.B. Seligman, Modular Lie Algebras, Springer-Verlag, Berlin, 1967. MR 39:6933

76. R. Steinberg, Representations of algebraic groups, Nagoya Math. J. 22 (1963), 33-56. MR 27:5870

77. H. Strade and R. Farnsteiner, Modular Lie Algebras and their Representations, Marcel Dekker, New York, 1988. MR 89h:17021

78. F.D. Veldkamp, The center of the universal enveloping algebra of a Lie algebra in characteristic p, Ann. Sci. École Norm. Sup. (4) 5 (1972), 217-240. MR 46:7341

79. D.-N. Verma, The rôle of affine Weyl groups in the representation theory of algebraic Chevalley groups and their Lie algebras, pp. 653-705, Lie Groups and Their Representations (Budapest, 1971), Halsted, New York, 1975. MR 53:13425

80. B.Yu. Weisfeiler and V.G. Kac, Irreducible representations of Lie p-algebras, Funktsional. Anal. i Prilozen. 5 (1971), no. 2, 28-36; English transl., Functional Anal. Appl. 5 (1971), 111-117. MR 44:2793

81. H. Zassenhaus, The representations of Lie algebras of prime characteristic, Proc. Glasgow Math. Assoc. 2 (1954), 1-36. MR 16:108c

Dept. of Mathematics \& Statistics, U. Massachusetts, Amherst, MA 01003-4515

E-mail address: jeh@math.umass.edu 